\title{
March 2014 Imaging Case of the Month
}

\author{
Michael B. Gotway, MD \\ Department of Radiology \\ Mayo Clinic Arizona \\ Scottsdale, AZ
}

Clinical History: A 60-year-old man with a history of severe asthmatic bronchitis presented with a 6 week history of intermittent fever, productive cough, shortness of breath, and decreased appetite. Four weeks earlier the patient was presumptively treated with amoxicillin for presumed community-acquired pneumonia, with some improvement, but his symptoms recurred 10 days following completion of his course of therapy. The patient also thought he was diagnosed with a COPD exacerbation during this time period and was treated with a short course of corticosteroids without improvement.

The patient was seen by his pulmonologist who noted decreased breath sounds over the right thorax, and referred the patient to the emergency room.

In the emergency room, a leukocytosis (white blood cell count $=17.4 \times 10^{9} / \mathrm{L}$ with neutrophilia) was noted. Broad-spectrum antibiotic coverage was re-instituted and frontal chest radiography (Figure 1) was performed.

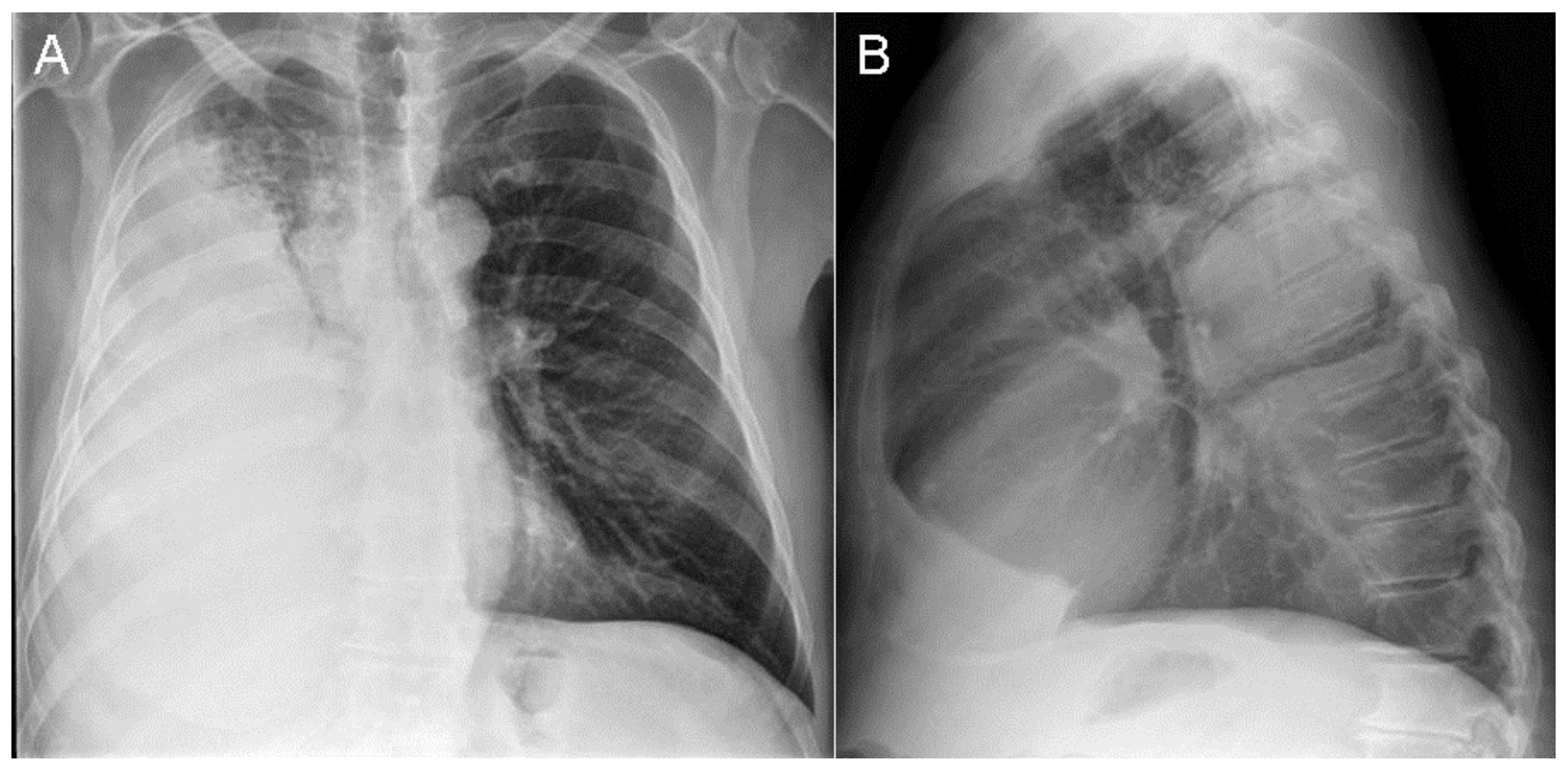

Figure 1: Frontal $(A)$ and lateral $(B)$ chest radiography 
Which of the following statements regarding the chest radiograph is most accurate? (Click on the correct answer to move to the next panel)

1. The chest radiograph homogeneous complete right lung consolidation

2. The chest radiograph shows a right-sided mediastinal mass

3. The chest radiograph shows homogeneous right lung opacity suggesting right lung collapse

4. The chest radiograph shows homogeneous right lung opacity suggesting a large right pleural effusion

5. The chest radiograph shows multiple nodules 


\section{Correct! \\ 4. The chest radiograph shows homogeneous right lung opacity suggesting a large right pleural effusion}

The chest radiograph shows fairly homogeneous opacity involving the right thorax, largely devoid of air bronchograms, and without significant volume loss- note that the trachea is positioned equidistant from each clavicle, when accounting for mild left anterior oblique patient positioning of the chest radiograph. If anything, minimal mass effect on the heart is noted, suggesting that the right thoracic process is spaceoccupying. Most of the right thoracic opacity is homogeneous without air bronchograms, suggesting that the process is extraparenchymal in location. When a space-occupying extraparenchymal process is present on a chest radiograph, an abnormality related to the pleura is the most likely consideration. The only clearly visible lung on the right side is present in the superior-medial right thorax. Some consolidation is present in this location, and lucencies are seen, suggesting that air-space disease is present in this location. However, most of the right lung process homogeneous and bland, without any features that suggest a parenchymal location [in particular, air bronchograms], which indicates that the right thoracic process originates from an extraparenchymal location. No nodules are present. The right-sided process has extensive contact with the right chest wall- far more so than the right mediastinum- which argues against a primary mediastinal location.

Which of the following is the least appropriate consideration among the differential diagnostic possibilities for the appearance of the patient's chest radiograph? (Click on the correct answer to move to the next panel)

1. Chest wall elastofibroma

2. Empyema

3. Hemothorax

4. Metastatic disease

5. Solitary fibrous tumor of the pleura 


\section{Correct! \\ 1. Chest wall elastofibroma}

All the choices provided are extraparenchymal lesions, but, among the possibilities listed, only choice " $e$ " does not arise from the pleura. Furthermore, chest wall elastofibromas typically arise posteriorly from the chest wall connective tissue, typically in the subscapular region, and are not detectable at chest radiography. All the other choices may produce a mass-like extraparenchymal process similar to what is seen at this patient's chest radiograph.).

The patient underwent thoracic ultrasound (Figure 2) for further characterization of the chest radiographic findings.

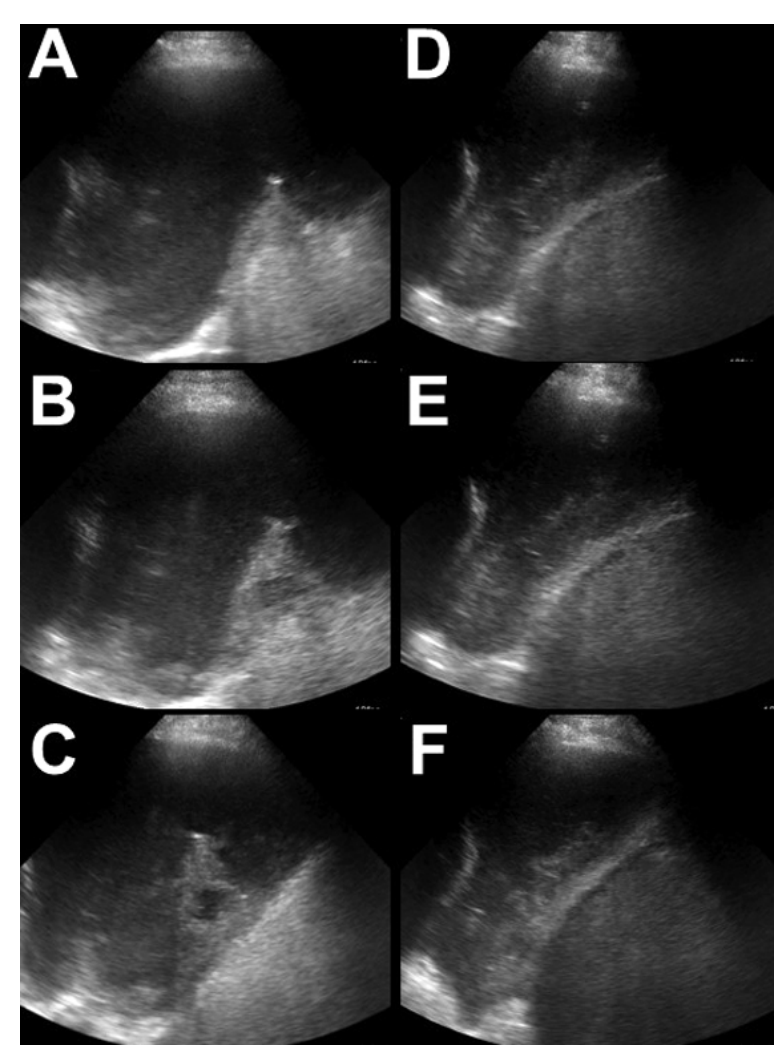

Figure 2. Longitudinal ultrasound images

Which of the following statements regarding this ultrasound is most accurate? (Click on the correct answer to move to the next panel)

1. The thoracic ultrasound shows a right hydropneumothorax

2. The thoracic ultrasound shows a right-sided diaphragmatic hernia

3. The thoracic ultrasound shows a solid right thoracic mass

4. The thoracic ultrasound shows complex right pleural fluid

5. The thoracic ultrasound shows only consolidated lung 


\section{Correct!}

\section{The thoracic ultrasound shows complex right pleural fluid}

The thoracic ultrasound shows mixed echogenicity right-sided pleural fluid (Figure 3).

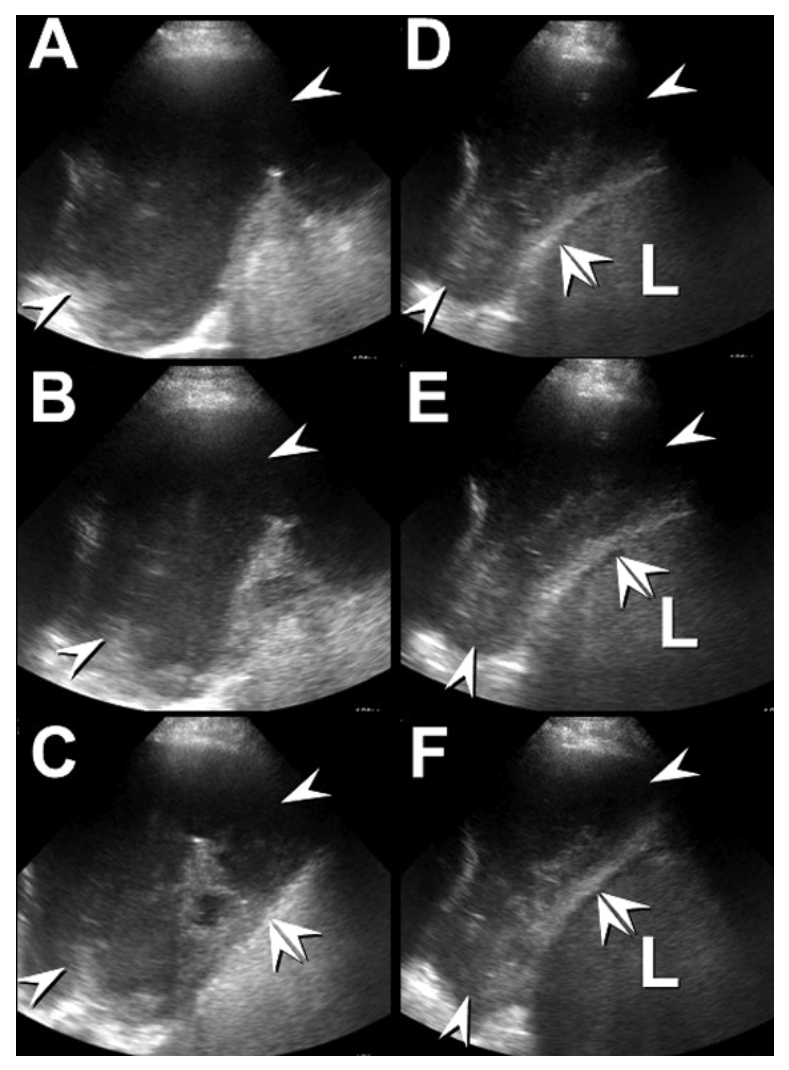

Figure 3. Serial longitudinal ultrasound images shows mixed echogenicity material (single arrowheads) superior to the right diaphragm (hyperechoic line indicated by double arrowheads). The liver $(L)$ is seen caudal to the diaphragm and right thorax.

The fluid is significantly complex. However, the material is not solid- note the areas of increased echogenicity posterior to the material, representing sound transmission, indicating the at least partial fluid nature of the material. The liver is clearly depicted caudal to an intact diaphragm, so there is no evidence to suggest diaphragmatic hernia. Furthermore, there are no features to suggest pleural space gas- such as ring-down artifact (the "comet-tail" sign).

What is the next most appropriate step in the management of this patient?

1. Bronchoscopy with bronchoalveolar lavage

2. Decubitus radiography

3. Start anti-fungal medications

4. Transthoracic echocardiography

5. Ultrasound-guided thoracentesis 


\section{Correct!}

\section{Ultrasound-guided thoracentesis}

Initiating a new course of antibiotic therapy is premature, given that broad-spectrum antibacterial medications have already been administered and nothing in the patient's presentation or imaging thus far strongly indicates the presence of a fungal infection. The process identified at chest radiography and ultrasound is primarily pleural in location, suggesting that bronchoscopy with bronchoalveolar lavage will be of little use. Some parenchymal lung disease may be present, but as yet requires more investigation before further invasive approaches are entertained. Transthoracic echocardiography would be of no value for the assessment of the right pleural space process. While decubitus radiography can be valuable for determining whether or not mobile pleural fluid is present, and deciding whether or not to attempt percutaneous sampling of any such fluid, this information has already been obtained with the transthoracic ultrasound procedure.

Right-sided transthoracic ultrasound was performed. Approximately $250 \mathrm{cc}$ of cloudy, yellow, slightly malodorous fluid was obtained from the right pleural space, but despite various maneuvers, no more fluid could be withdrawn owing to the complex, viscous nature of the fluid.

What of the following is the most appropriate next step?

1. Bronchoscopy with bronchoalveolar lavage

2. Decubitus radiography

3. Obtain thoracic CT

4. Perform thoracic MRI

5. Repeat chest radiography 


\section{Correct! \\ 3. Obtain thoracic CT}

The results of the ultrasound procedure suggest that the right pleural space process is complex and multiloculated, and it is likely further intervention will be required. Thoracic $\mathrm{CT}$ is the optimal method for further non-invasive investigation of the right pleural space process. Repeating the chest radiograph is not an "incorrect" maneuver, given that radiography is often performed after a pleural space intervention to exclude a complication such as pneumothorax. However, such a complication is very unlikely given the large right pleural space abnormality and minimal amount of fluid removed. Furthermore, thoracic CT will be quite capable of excluding post-procedural pneumothorax while simultaneously providing additional information regarding the right pleural space process. Similar to upright frontal chest radiography, decubitus chest radiography will not provide additional useful information at this point in the patient's course. Bronchoscopy with bronchoalveolar lavage is again not the procedure of choice at this point. Thoracic MRI could provide information regarding the pleural space process similar, but not superior to, thoracic $\mathrm{CT}$, and typically costs more and takes longer to perform than thoracic $\mathrm{CT}$, and is incapable of providing information equivalent to thoracic CT regarding the status of the lung parenchyma.

Thoracic CT (Figure 4) was performed.

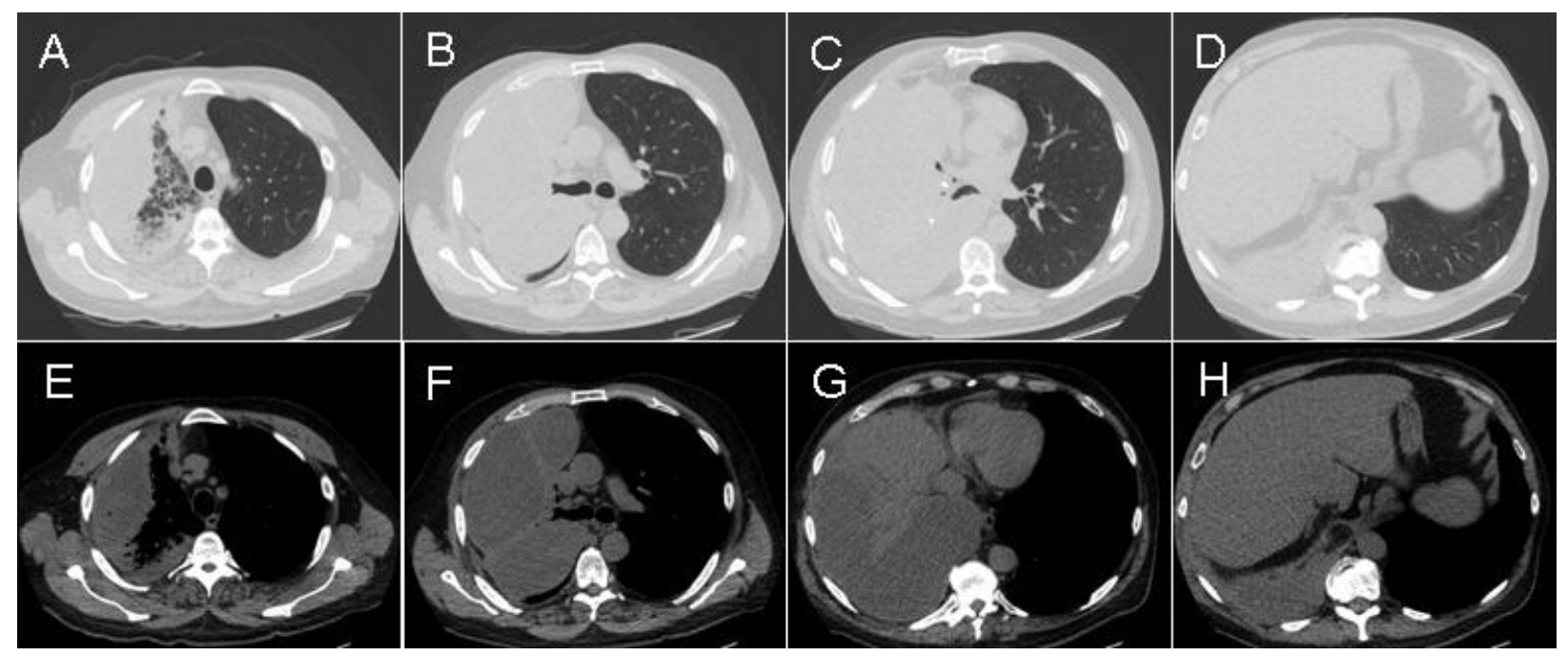

Figure 4. Panels A-D: Representative thoracic CT lung windows. Panels E-H: Corresponding soft tissue windows.

Which of the following statements regarding these CT examinations is most accurate? 
1. The thoracic CT shows a complex mass in the right thorax that arises from the ribs and chest wall soft tissues, not the pleural space

2. The thoracic CT shows a multiloculated process which actually resides in the lung parenchyma, not the pleural space

3. The thoracic CT shows a multiloculated right pleural space collection suggesting the diagnosis of empyema

4. The thoracic CT shows a right pleural space mass suggesting a primary pleural tumors

5. The thoracic CT shows large fluid-attenuation mass arising from the mediastinum 


\section{Correct! \\ 3. The thoracic CT shows a multiloculated right pleural space collection suggesting the diagnosis of empyema}

The thoracic CT shows a complex right-sided fluid collection creating obtuse angles with the chest wall, indicating an extraparenchymal origin. The complex fluid is peripherally positioned, which is inconsistent with a primary mediastinal origin. There is no rib destruction or chest wall mass to suggest the process arises from these structures; rather, the appearance is consistent with a pleural space origin. A small focus of gas seen superiorly is consistent with the recent ultrasound-guided thoracentesis. Although the thoracic CT was performed without intravenous contrast, peripheral smooth parietal pleural thickening is evident. Extensive medial right lung consolidation is present. The appearance of a multiloculated pleural space collection with smooth pleural thickening is highly suggestive of empyema.

Streptococcus intermedius was recovered from the right-sided ultrasound-guided thoracentesis. The patient subsequently underwent surgical decortication. One pleural fluid loculation remained along the anteromedial right thorax following the surgical procedure, and was successfully drained percutaneously. The patient subsequently recovered uneventfully.

Diagnosis: Empyema secondary to Streptococcus intermedius

\section{References}

1. Leung AN, Müller NL, Miller RR. CT in differential diagnosis of diffuse pleural disease. AJR Am J Roentgenol. 1990; 154(3):487-492. [CrossRef] [PubMed]

2. Aquino SL, Webb WR, Gushiken BJ. Pleural exudates and transudates: diagnosis with contrast-enhanced CT. Radiology. 1994; 192(3):803-808. [PubMed] 